\title{
A MODEL-INDEPENDENT DETERMINATION OF THE EXPANSION AND ACCELERATION RATES OF THE UNIVERSE AS A FUNCTION OF REDSHIFT AND CONSTRAINTS ON DARK ENERGY
}

\author{
Ruth A. DALY \\ Department of Physics, Berks-Lehigh Valley College, Pennsylvania State University, Reading, PA 19610; \\ rdaly@psu.edu \\ AND \\ S. G. DJORGOVSKI \\ Division of Physics, Mathematics, and Astronomy, California Institute of Technology, MS 105-24, Pasadena, CA 91125; \\ george@astro.caltech.edu \\ Received 2003 April 3; accepted 2003 July 3
}

\begin{abstract}
Determination of the expansion and acceleration history of the universe is one of the fundamental goals of cosmology. Detailed measurements of these rates as a function of redshift can provide new physical insights into the nature and evolution of the dark energy, which apparently dominates the global dynamics of the universe at the present epoch. We present here dimensionless coordinate distances $y(z)$ to 20 radio galaxies reaching out to $z \approx 1.8$, the redshift range currently not covered by supernova standard-candle observations. There is very good agreement between coordinate distances to radio galaxies and supernovae for the redshift range in which these measurements overlap, suggesting that neither is plagued at this level by unknown systematic errors. We develop a simple numerical method for a direct determination of the expansion and acceleration rates, $E(z)$ and $q(z)$, from the data, which makes no assumptions about the underlying cosmological model or the equation of state parameter $w$. This differential method is in contrast to the traditional cosmological tests, in which particular model equations are integrated and then compared with the observations. The new approach is model independent, but at a cost of being noisier and highly sensitive to the amount and quality of the available data. We illustrate the method by applying it to the currently available supernova data and the data on radio galaxies presented here. We derive the expansion rate of the universe as a function of redshift, $E(z)$, and for the first time we obtain a direct estimate of the acceleration rate of the universe as a function of redshift, $q(z)$, in a way that is independent of assumptions regarding the dark energy and its redshift evolution. The current observations indicate that the universe makes a transition from acceleration to deceleration at a redshift greater than 0.3 , with a best-fit estimate of about 0.45 ; this transition redshift and our determinations of $E(z)$ are broadly in agreement with the currently popular Friedmann-Lemaitre cosmology with $\Omega_{m}=0.3$ and $\Omega_{\Lambda}=0.7$, even though no model assumptions are made in deriving the fits for $E(z)$ and $q(z)$. With the advent of much better and richer data sets in the future, our direct method can provide a useful complementarity and an independent check on the traditional cosmological tests.
\end{abstract}

Subject headings: cosmological parameters — cosmology: observations — cosmology: theory — dark matter - equation of state

\section{INTRODUCTION}

A traditional task of cosmology is to determine the global geometry and dynamics of the universe. The field has been revolutionized by the modern measurements of cosmic microwave background (CMB) fluctuations (e.g., Bennett et al. 2003; Spergel et al. 2003, and references therein), the use of distant supernovae ( $\mathrm{SNe}$ ) in a Hubble diagram (see, e.g., Riess 2000; Leibundgut 2001, and references therein), radio galaxies (e.g., Daly \& Guerra 2002), and many other advances. What these modern measurements have now convincingly demonstrated is that the global mass-energy budget of the universe, and thus its dynamics, is dominated by a so-called dark energy component, which accounts for $\gtrsim 70 \%$ of the closure density today. The Einstein cosmological constant, $\Lambda$, is one special (and viable) case. More generally, this mysterious dark energy component is characterized through the equation of state, $w=p / \rho$, where $p$ is the pressure and $\rho$ the energy density; the cosmological constant solution corresponds to $w=-1$. For reviews and further references, see, for example, Sahni \& Starobinsky
(2000), Turner (2002, 2003), Peebles \& Ratra (2003), and Padmanabhan (2003).

The nature of the dark energy (including its evolution in redshift, if any) is one of the most outstanding problems of physics and astronomy today. Constraining it through analysis of cosmological data is a task of a critical importance, and every new data set or analysis method can provide valuable insights into this problem.

Several recent studies have focused on the use of supernovae to determine the properties of the dark energy (Starobinsky 1998; Huterer \& Turner 1999; Saini et al. 2000; Chiba \& Nakamura 2000; Maor, Brustein, \& Steinhardt 2001; Goliath et al. 2001; Astier 2001; Gerke \& Efstathiou 2002; Weller \& Albrecht 2002; Padmanabhan \& Choudhury 2002). The key ingredients are luminosity distances to sources over a broad range of redshift, preferably including sources at high redshift. Most of these analyses have focused on constraints on an evolving scalar field such as that used to define quintessence (Caldwell, Dave, \& Steinhardt 1998) or a rolling scalar field (Peebles \& Ratra 1988). More recently, other types of models have been proposed to 
account for the acceleration of the universe, such as stringy dark energy (Frampton 2003) and $k$-essence (ArmendarizPicon, Damour, \& Mukhanov 1999; Barger \& Marfatia 2001).

Here we focus on direct empirical determinations of the dimensionless expansion rate $E(z)$ and acceleration rate $q(z)$ as functions of redshift. These require values for dimensionless coordinate distances to sources over a broad range of redshifts. We provide both a new data set and a new method for estimating $E(z)$ and $q(z)$.

We first present coordinate distances to 20 radio galaxies (RGs), reaching out to $z \approx 1.8$, and thus supplementing the existing SN data in what is a critical redshift regime. These RG data can be used to compare model predictions of any flavor of dark energy with the observations. These coordinate distances are derived and listed in $\S 2$. For completeness and to compare the RG and supernova $\mathrm{SN}$ results, the coordinate distances to $78 \mathrm{SNe}$ are also listed in $\S 2$. In $\S 3$, we derive the expressions for a direct determination of $E(z)$ and $q(z)$ from measurements of the dimensionless coordinate distances $y(z)$. In $\S 4$, we describe our simple numerical differentiation technique, which can be used to implement these concepts on the real data. We illustrate the method and present our preliminary results based on the current RG and SN data sets in $\S 5$ and discuss implications for the properties of the dark energy in $\S 6$. A summary and discussion follows in $\S 7$.

\section{DIMENSIONLESS COORDINATE DISTANCES}

The values of coordinate distances to sources at high redshift can be used to determine or constrain global cosmological parameters and to understand the properties and redshift evolution of the dark energy. Coordinate distances $\left(a_{0} r\right)$ may be obtained from luminosity distances $d_{L}$ or angular size distances $d_{A}$, since these are simply related to the coordinate distance: $d_{L}=\left(a_{0} r\right)(1+z)$ and $d_{A}=\left(a_{0} r\right) /$ $(1+z)$ (e.g., Weinberg 1972).

The dimensionless coordinate distance, $y(z)$, is simply related to the coordinate distance $a_{0} r$ by $y(z)=H_{0}\left(a_{0} r\right)$ (e.g., Carroll, Press, \& Turner 1992; Peebles 1993). The luminosity distance $d_{L}$ and the angular size distance $d_{A}$ are also simply related to the dimensionless coordinate distance: $d_{L}=H_{0}^{-1} y(z)(1+z)=H_{0}^{-1} D_{L}$, where $D_{L}$ is the dimensionless luminosity distance (e.g., Perlmutter et al. 1999) and $d_{A}=H_{0}^{-1} y(z) /(1+z)$. Observations of Type Ia supernovae and type IIb radio galaxies allow estimates of the dimensionless coordinate distances to sources at different redshifts.

The use of FR IIb radio galaxies to determine the angular size distance or coordinate distance to radio galaxies at different redshifts is described in detail elsewhere (e.g., Podariu et al. 2003; Daly \& Guerra 2002; Guerra, Daly, \& Wan 2000; Daly 1994; Guerra \& Daly 1998). In addition to the use of FR IIb radio galaxies addressed here, other methods of using radio galaxies and quasars to determine coordinate distances are discussed by Buchalter et al. (1998), Gurvits, Kellermann, \& Frey (1999), Vishwakarma (2001), Lima \& Alcaniz (2002), and Chen \& Ratra (2003). Here FR IIb radio galaxies are used to obtain dimensionless coordinate distances to 20 radio galaxies by following the method described, for example, by Daly \& Guerra (2002).

In the radio galaxy method proposed by Daly (1994), one model parameter $\beta$ enters into the ratio $R_{*} \equiv\langle D\rangle / D_{*}$; this
TABLE 1

Radio Galaxy Dimensionless Coordinate Distances

\begin{tabular}{|c|c|c|c|c|c|}
\hline Source & Redshift & $y_{j}$ & $\sigma\left(y_{j}\right)$ & $y_{s}$ & $\sigma\left(y_{s}\right)$ \\
\hline 3C 405................. & 0.056 & 0.056 & 0.010 & 0.057 & 0.011 \\
\hline 3C 244.1 ................ & 0.430 & 0.445 & 0.071 & 0.462 & 0.079 \\
\hline 3C 330 .................. & 0.549 & 0.400 & 0.066 & 0.431 & 0.076 \\
\hline 3C 427.1 .................. & 0.572 & 0.319 & 0.051 & 0.319 & 0.054 \\
\hline 3C 337.................... & 0.630 & 0.600 & 0.071 & 0.630 & 0.080 \\
\hline 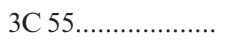 & 0.720 & 0.606 & 0.071 & 0.680 & 0.085 \\
\hline 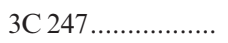 & 0.749 & 0.625 & 0.069 & 0.660 & 0.077 \\
\hline $3 \mathrm{C} 265 \ldots \ldots$ & 0.811 & 0.667 & 0.081 & 0.731 & 0.093 \\
\hline $3 C 325 \ldots \ldots$ & 0.860 & 0.818 & 0.149 & 0.885 & 0.162 \\
\hline 3C 289.................. & 0.967 & 0.681 & 0.108 & 0.722 & 0.122 \\
\hline 3C 268.1 .................. & 0.974 & 0.780 & 0.127 & 0.855 & 0.149 \\
\hline 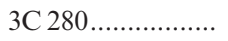 & 0.996 & 0.703 & 0.111 & 0.758 & 0.128 \\
\hline 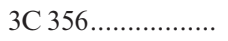 & 1.079 & 0.842 & 0.151 & 0.979 & 0.188 \\
\hline 3C $267 \ldots \ldots \ldots \ldots \ldots$ & 1.144 & 0.753 & 0.126 & 0.837 & 0.150 \\
\hline 3C 194_................ & 1.190 & 1.141 & 0.205 & 1.251 & 0.239 \\
\hline 3C 324................... & 1.210 & 0.996 & 0.251 & 1.081 & 0.291 \\
\hline 3C 437................. & 1.480 & 0.849 & 0.206 & 0.992 & 0.260 \\
\hline $3 \mathrm{C} 68.2 \ldots$ & 1.575 & 1.477 & 0.386 & 1.717 & 0.484 \\
\hline $3 C 322 \ldots$ & 1.681 & 1.167 & 0.249 & 1.356 & 0.316 \\
\hline 3C 239.................. & 1.790 & 1.246 & 0.257 & 1.419 & 0.318 \\
\hline
\end{tabular}

ratio also depends on observed quantities and the dimensionless coordinate distance $y(z)$. In this model the ratio $R_{*}$ is equal to a constant, $\kappa$ :

$$
R_{*}[\beta, y(z)]=\kappa .
$$

The constants $\kappa$ and $\beta$ and their uncertainties are obtained by fitting all the data to equation (1), as described in detail by Guerra et al. (2000), and Daly \& Guerra (2002). The ratio is given by

$$
R_{*}=k_{0} y^{(6 \beta-1) / 7}\left(k_{1} y^{-4 / 7}+k_{2}\right)^{\beta / 3-1},
$$

where $k_{0}, k_{1}$, and $k_{2}$ are observed quantities (described in detail in the Appendix of Guerra et al. 2000). Equation (1) with $R_{*}$ given by equation (2) allows a determination of $y(z)$ to each source; $y(z)$ is implicitly known for each source and is determined using an iterative technique. The values of $y$ obtained along with the $1 \sigma$ error of $y$ are listed in Table 1. In determining the $1 \sigma$ error bar on $y(z)$, the uncertainties of $\kappa, k_{0}, k_{1}$, and $\beta$ have been included; $k_{2}$ is known to high precision as it is the energy density of the microwave background radiation at the source redshift and is the term that describes the effects of inverse Compton cooling of relativistic electrons by the microwave background radiation. The best-fit values of $\kappa$ and $\beta$ vary slightly, depending on whether just the radio galaxy data are fitted or both the radio galaxy and supernova data are fitted. Values of $y$ obtained using the best-fit parameters to radio galaxies alone are labeled $y_{s}$ in Table 1, and those obtained using the best fit to both the radio galaxy and supernova data are labeled $y_{j}$; that is, the best-fit values of $\kappa$ and $\beta$ change slightly, depending on whether just the radio galaxy data are fitted (marked with subscript $s$ ) or the radio galaxy and supernovae data are fitted simultaneously (marked with subscript $j$ ). Best-fit values of $\kappa$ and $\beta$ are listed in Table 2 . Note that the radio galaxy method does not rely on a lowredshift normalization; the best-fit values of $\kappa$ and $\beta$ are determined using all the data. 
TABLE 2

Best-Fit Parameters

\begin{tabular}{|c|c|c|c|c|c|c|}
\hline Model & Fit To & $\mathscr{M}_{B}$ & $\kappa$ & $\beta$ & $\chi^{2} / \operatorname{dof}(\mathrm{SN})$ & $\chi^{2} / \operatorname{dof}(\mathrm{RG})$ \\
\hline Q & SN/RG only (s) & $23.91 \pm 0.03$ & $8.88 \pm 0.05$ & $1.70 \pm 0.04$ & $56.2 / 50$ & $16.53 / 16$ \\
\hline Q & $\mathrm{SN}+\mathrm{RG}(\mathrm{j})$ & $23.95 \pm 0.03$ & $8.81 \pm 0.05$ & $1.75 \pm 0.04$ & $74.1 / 68$ & $74.1 / 68$ \\
\hline $\mathrm{SF} \ldots \ldots \ldots \ldots \ldots \ldots \ldots \ldots \ldots \ldots$ & $\mathrm{SN} / \mathrm{RG}$ only (s) & $23.94 \pm 0.03$ & $8.90 \pm 0.05$ & $1.70 \pm 0.04$ & $56.7 / 50$ & $16.7 / 16$ \\
\hline SF . ....................... & $\mathrm{SN}+\mathrm{RG}(\mathrm{j})$ & $23.95 \pm 0.03$ & $8.81 \pm 0.05$ & $1.80 \pm 0.03$ & $74.1 / 68$ & $74.1 / 68$ \\
\hline
\end{tabular}

Note.- Values obtained from the fits of Daly \& Guerra (2002) that allow for quintessence in a spatially flat universe with separate (s) and joint (j) fits to the radio galaxy and supernovae data are labeled "Q." Best-fit values obtained in the rolling scalar field model analyzed by Podariu et al. 2003 are labeled "SF."

The best-fit values of $\kappa, \beta$, and $\mathscr{M}_{B}$ (described below) and their error bars are included in Table 2, in which the 54 supernovae included in the "primary fit C" of Perlmutter et al. (1999) and the 20 radio galaxies discussed here were studied. Values obtained from the fits of Daly \& Guerra (2002) that allow for quintessence in a spatially flat universe with separate (s) and joint (j) fits to the radio galaxy and supernovae data are labeled " Q." Best-fit values obtained in the rolling scalar field model analyzed by Podariu et al. (2003) are labeled "SF." As the number of data points in the fit increases, the value of each constant, $\kappa, \beta$, and $\mathscr{M}_{B}$, becomes independent of the assumptions of the fit. For example, the 54 supernovae points yield a consistent value of $\mathscr{M}_{B}$ for fits that include supernovae only or radio galaxies and supernovae and in a universe with quintessence or a rolling scalar field. Since the value of $\mathscr{M}_{B}$ changes so little when fitted in different models and when fitted by including or excluding radio galaxies, only one value of $y$ is listed for each supernova. The 20 radio galaxy points show some small variations in the values of $\kappa$ and $\beta$ obtained with radio galaxies alone or radio galaxies and supernovae obtained in a universe with quintessence or a rolling scalar field. As more radio galaxy data points are added, the values of the constants will be more accurately determined. New runs were done that include the full 78 supernovae listed here and the best-fit parameters, and their error bars are the same as those listed in Table 2.

The coordinate distances to the supernovae are determined following the procedures of Perlmutter et al. (1999) and Riess et al. (1998). In the application of supernovae Type Ia as a distance indicator, there is one model parameter, $\alpha$, which is used to determine the effective apparent $B$-band magnitude at maximum brightness, $m_{B}^{\text {eff }}$. This is related to the dimensionless coordinate distance $y(z)$ :

$$
m_{B}^{\text {eff }}(\alpha)=\mathscr{M}_{B}+5 \log [(1+z) y(z)] .
$$

The constant $\mathscr{M}_{B}$ is determined by fitting all the supernova data and is simply related to the absolute magnitude of the supernova peak brightness $M_{B}: \mathscr{M}_{B}=M_{B}+25-5 \log H_{0}$ (see Perlmutter et al. 1999). Equation (3) is then used to determine $y(z)$ to each of the 54 supernovae in the " primary fit C" of Perlmutter et al. (1999), the 37 supernovae presented by Riess et al. (1998), and the one high-redshift supernova published by Riess et al. (2001), with the magnitude of this source corrected for gravitational lensing (Benitez et al. 2002). The $1 \sigma$ uncertainty of y is obtained by combining the uncertainties of $\mathscr{M}_{B}$ and $m_{B}^{\mathrm{eff}}$. These values of listed in Table 2. There are 14 sources that are present in both the Riess et al. (1998) and Perlmutter et al. (1999) samples used here. In the determinations of $E(z)$ and $q(z)$, average values of $y$ with appropriate error bars were used for these duplicate sources; these values are listed in Table 4 . The values of $y(z)$ are shown in Figures 1 and 2. The good agreement between coordinate distances determined using radio galaxies and supernovae at similar redshifts is easy to see in these figures.

To test the reliability of the values of $y(z)$ obtained for the radio galaxies, a comparison was made between cosmological parameters obtained directly from the radio galaxy data alone and those obtained from the values of $y_{s}$ listed in Table 1 in a quintessence model (see line 1 of Table 2). Each value of $y_{s}$ was substituted into equation (3) to obtain an equivalent effective apparent magnitude for the radio galaxy; the value of $\mathscr{M}_{B}$ obtained for supernovae alone in a universe with quintessence (line 1 of Table 2) was adopted. These effective apparent magnitudes were then analyzed in a universe with quintessence and the best-fit parameters and their $1 \sigma$ ranges compared with those obtained directly from

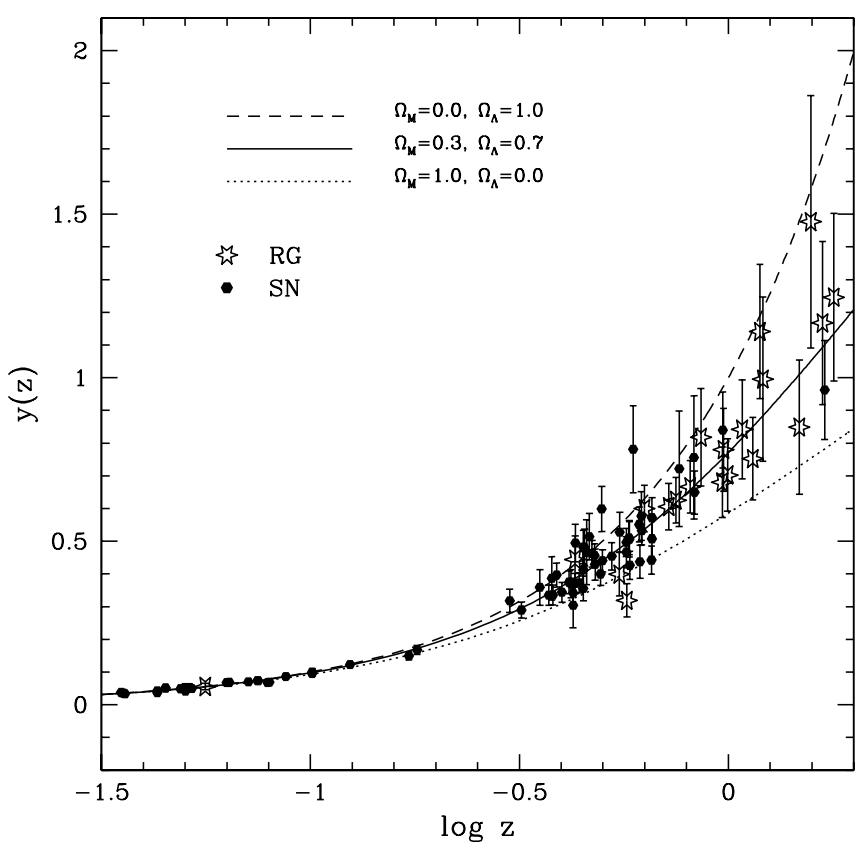

FIG. 1.-Dimensionless coordinate distances $y(z)$ to 20 radio galaxies and 78 supernovae as a function of $\log z$. Note that the determinations of $y(z)$ have been made using the best-fit value of $\mathscr{M}_{B}$ obtained for the full data set of 78 supernovae and 20 radio galaxies, and the best-fit value of $\kappa$ obtained using the full data set ( $y_{j}$ for radio galaxies). Radio galaxies are shown as open stars, and supernovae are shown as filled circles. Very similar results obtain when values of $y_{s}$ for radio galaxies are shown, as in Fig. 2. 


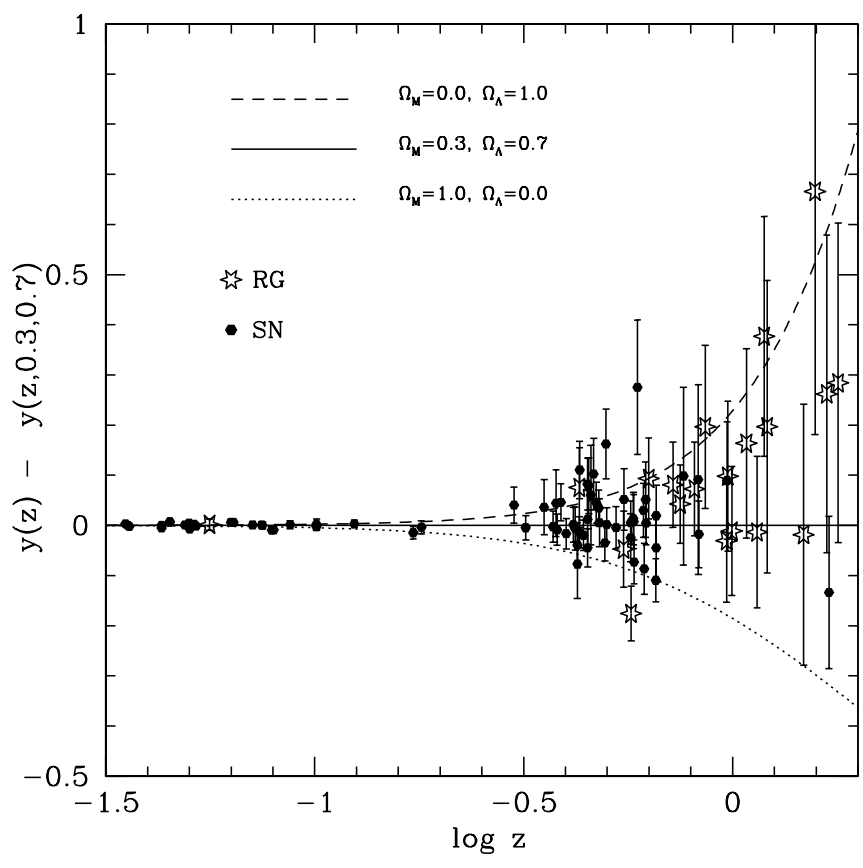

FIG. 2.-Residuals between $y(z)$ and those expected in a universe with $\Omega_{m}=0.3$ and $\Omega_{\Lambda}=0.7$, where $y(z)$ is the dimensionless coordinate distance, shown as a function of $\log z$. Values of $y_{s}$, obtained using the best-fit values of $\kappa$ and $\beta$ determined using radio galaxies alone, are shown. The results obtained when best-fit values to the full data set are used $\left(y_{j}\right.$ for radio galaxies) are very similar, as shown in Fig. 1. Radio galaxies are shown as open stars, and supernovae are shown as filled circles.

the radio galaxy data. First, the $\chi^{2}$ per degree of freedom went from $16.5 / 16$ to $15.6 / 15$, so the reduced $\chi^{2}$ remains fairly constant; the number of degrees of freedom drops by 1 in the new fit since one new parameter, $\mathscr{M}_{B}$ is fitted. The 1 $\sigma$ range of $\Omega_{m}$ is $0.0-0.24$ in the original fit and $0.0-0.17$ in the new fit. The $1 \sigma$ range of $\Omega_{Q}$ is $0.76-1.0$ in the original fit and $0.83-1.0$ in the new fit. The $1 \sigma$ range of $w$ in the original fit is -1.3 to -0.43 centered on -0.73 , and -1.5 to -0.56 centered on 0.8 in the new fit. The best-fit value of $\mathscr{M}_{B}$ is $23.83 \pm 0.08$, compared with the input value $\mathscr{M}_{B}$ of $23.91 \pm 0.03$ used to define an effective apparent magnitude for each radio galaxy; thus, the cosmological parameters obtained directly from the radio galaxies are very similar to those obtained from the values of $y(z)$ listed in Table 1.

\section{COMPUTATION OF $E(z)$ AND $q(z)$ FROM THE COORDINATE DISTANCES}

The determinations of the dimensionless coordinate distances do not require any assumptions regarding cosmological parameters, the dark energy, or the redshift evolution of these components once the values of the constants $\kappa, \beta$, and $\mathscr{M}_{B}$ have been determined. The first and second derivatives of the dimensionless coordinate distance with respect to redshift can be used to construct a model-independent determination of the dimensionless expansion rate $E(z)=H(z) / H_{0}$ and the acceleration rate $q(z)=-\ddot{a} a / \dot{a}^{2}$.

These follow from the relation between redshift $z$ and the cosmic scale factor $a(t), a(t) / a_{0}=(1+z)^{-1}$, and the Robertson-Walker line element, which describes a homogeneous isotropic expanding universe,

$$
d \tau^{2}=d t^{2}-a^{2}(t)\left(\frac{d r^{2}}{1-k r^{2}}+r^{2} d \theta^{2}+r^{2} \sin ^{2} \theta d \phi^{2}\right)
$$

(see, e.g., Weinberg 1972). It is well known that these imply $H(z) \equiv \dot{a} / a=\left(1-k r^{2}\right)^{1 / 2}(d y / d z)^{-1} H_{0}$, and with $k=0$ and $H(z)=H_{0} E(z)$,

$$
E(z)=(d y / d z)^{-1}
$$

(e.g., Weinberg 1972; Peebles 1993). Recent CMB measurements indicate that our universe has zero space curvature, $k=0$ (e.g., Bennett et al. 2003; Spergel et al. 2003); thus in principle the data $y(z)$ can be used to empirically determine the $d y / d z$ and the dimensionless expansion rate $E(z)$. This, in turn, is related to cosmological parameters such as dark energy and their redshift evolution as discussed in $\S 6$. For example, in a universe with quintessence (Caldwell et al. 1998), which has a time-independent equation of state $w=P / \rho, \quad E^{2}(z)=\sum \Omega_{i}(1+z)^{n_{i}}$, where $w_{i}=P_{i} / \rho_{i}$ and $n_{i}=3\left(1+w_{i}\right)$ (see, e.g., Turner \& White 1997; Peebles \& Ratra 2003; Daly \& Guerra 2002). The deceleration parameter at the present epoch is $q_{0}=-(\ddot{a} a / \dot{a})_{0}=$ $0.5 \sum \Omega_{i}\left(1+3 w_{i}\right)$, when $w_{i}$ is time independent.

A direct empirical determination of the acceleration of the universe as a function of redshift can be obtained from $y(z)$ without making any assumptions about the nature or evolution of the "dark energy." This can be done using the equation

$$
-q(z) \equiv \frac{\ddot{a} a}{\dot{a}^{2}}=1+(1+z)\left(\frac{d y}{d z}\right)^{-1} \frac{d^{2} y}{d z^{2}}
$$

(Daly 2002), valid for $k=0$; if $k \neq 0$, another term, $\left[k r(1+z) /\left(1-k r^{2}\right)\right] d r / d z$, must be added to the right-hand side of equation (6).

Equation (6) depends only on the Robertson-Walker line element and the relation $1+z=a_{0} / a(t)$; thus, this expression for $q(z)$ is valid for any homogeneous isotropic expanding universe in which $1+z=a_{0} / a(t)$ and is consequently quite general and can be compared with any model to account for the acceleration of the universe, as long as the model describes a homogeneous isotropic expanding universe with the standard relation between $z$ and $a(t)$. The Robertson-Walker line element is given by equation (4). A light ray emitted by a galaxy traveling to us along the radial coordinate $r$ has $d \tau=d \theta=d \phi=0$. The increment is along the negative direction of $d r$, so equation (4) with $k=0$ implies that $a_{0} d r=-(1+z) d t \quad$ or $\quad d z / d t=$ $-a_{0}^{-1}(1+z)(d r / d z)^{-1}$. Differentiating $1+z=a_{0} / a(t)$ with respect to time implies that $\dot{a}=-a_{0}(1+z)^{-2} d z / d t$. Substituting for $d z / d t$, we find $\dot{a}=(1+z)^{-1}(d r / d z)^{-1}$. Differentiating again with respect to time, we find

$\ddot{a}=-(1+z)^{-2} \frac{d z}{d t}\left(\frac{d r}{d z}\right)^{-1}\left[1+(1+z)\left(\frac{d r}{d z}\right)^{-1} \frac{d^{2} r}{d z^{2}}\right]$,

which simplifies to equation (6) by using the expressions given here and the relation $y(z)=H_{0}\left(a_{0} r\right)$. For $k \neq 0$, equation (4) implies that $a_{0} d r=-(1+z)\left(1-k r^{2}\right)^{1 / 2} d t$ or $d z / d t=-a_{0}^{-1}(1+z)\left(1-k r^{2}\right)^{1 / 2}(d r / d z)^{-1}$. Differentiating $1+z=a_{0} / a(t)$ with respect to time implies that $\dot{a}=-a_{0}(1+z)^{-2} d z / d t$. Substituting for $d z / d t$, we find $\dot{a}=(1+z)^{-1}\left(1-k r^{2}\right)^{1 / 2}(d r / d z)^{-1}$. Differentiating again 
with respect to time, we find

$$
\begin{aligned}
\ddot{a}= & \left(a_{0}\right)^{-1}(1+z)^{-1}\left(1-k r^{2}\right)\left(\frac{d r}{d z}\right)^{-2} \\
& \times\left[1+(1+z)\left(\frac{d r}{d z}\right)^{-1} \frac{d^{2} r}{d z^{2}}\right]+\left(a_{0}\right)^{-1} k r\left(\frac{d r}{d z}\right)^{-1},
\end{aligned}
$$

thus

$$
\begin{aligned}
-q(z)= & \frac{\ddot{a} a}{\dot{a}^{2}}=1+(1+z)\left(\frac{d r}{d z}\right)^{-1} \frac{d^{2} r}{d z^{2}} \\
& +k r(1+z)\left(1-k r^{2}\right)^{-1} \frac{d r}{d z} .
\end{aligned}
$$

Equation (6) can in principle be used to empirically determine the redshift at which the universe makes a transition from acceleration to deceleration without requiring assumptions regarding the nature and redshift evolution of the " dark energy." The supernova and radio galaxy data allow a determination of the dimensionless coordinate distance $y$ to each source at redshift $z$. These data can then be used to determine $d y / d z$, and $d^{2} y / d z^{2}$; these can then be substituted into equation (6) to determine $q(z)$.

Since equations (5) and (6) are obtained without any assumptions regarding the mass-energy components of the universe or their redshift evolution, they can be used to directly determine the dimensionless acceleration rate $E(z)=(d y / d z)^{-1}$, which contains important information on the "dark energy" and its redshift evolution, and to determine the dimensionless acceleration parameter $q(z)$ directly from measurements of $y(z)$.

In the determinations of $y(z)$ a value of $\mathscr{M}_{B}$ must be adopted for the supernovae (see eq. [3]), and a value of $\kappa$ must be adopted for radio galaxies (see eq. [1]). These are not determined as a normalization by using only lowredshift sources. They are determined by fitting all the data and solving for the best-fit values of these parameters. Fits to the supernovae data, the radio galaxy data, and the joint data set were run for a variety of cases (see Table 2 ), such as a universe with quintessence $(Q)$ or a rolling scalar field (SF). There are enough supernovae that the value of $\mathscr{M}_{B}$ changes very little for different fits to the supernovae data, and they change very little if the supernovae data are considered separately or in conjunction with the radio galaxy data; thus, values of $y$ for supernovae do not change with the data set or model considered. The radio galaxy data best-fit parameters for $\kappa$ and $\beta$ change slightly depending on whether just the radio galaxies are considered or whether the full data set of radio galaxies plus supernovae are included. Values of $y_{s}$ obtained for the best-fit value of $\kappa$ by using radio galaxies alone in a universe with quintessence are listed, as well as the values $y_{j}$ obtained by using the best-fit values of $\kappa$ and $\beta$ for fits to the full data set of radio galaxies and supernovae. These values are listed in Table 1 and are considered and compared in the analyses of $E(z)$ and $q(z)$.

\section{NUMERICAL DIFFERENTIATION TECHNIQUE}

The key problem in this approach, of course, is that it requires a numerical differentiation of typically noisy data, which is a cardinal sin for any empirical scientist. This, after all, is the reason why all standard cosmological tests (e.g., the Hubble diagram) consist of integrating the model equations to compare them with the measurements. An additional problem is posed by the sparse and/or uneven coverage of the redshift range(s) of interest. While a numerical differentiation of noisy data is in general not advisable, it is certainly possible, and if done properly (in a statistical sense), it can produce meaningful results within the limits of the available data.

Most numerical differentiation techniques explicitly or implicitly assume that the data can be locally represented by some smooth (differentiable) function, whose derivative is then defined analytically. Typically this local approximation is a low-order polynomial; thus, estimation of derivatives is coupled in a self-consistent way with the estimation of the function representing the data themselves. Measurement errors can then be propagated in the standard manner, leading to estimated uncertainties of the fitted function values, as well as the derivatives. In our case, the function to be approximated, along with its first and second derivatives, is the dimensionless coordinate distance as a function of redshift, $y(z)$. The situation is simplified by the fact that the errors in $z$ are negligible compared with the errors in $y$, and thus the ordinary least-squares approach can be used.

There are three sources of errors when evaluating any function fits to noisy finite data sets. First, the errors of the individual data points: the least-squares approach deals with them in a statistically optimal fashion, provided that the quoted error bars are truly representative and that the deviations from the "true" underlying trend are drawn from a normal distribution. Second, if the fitted function is not a good approximation to the true trend, the results may be systematically biased. Locally, any function can be approximated as a polynomial (or as a Taylor series), and this becomes an issue of a having a sufficiently high fit order to account for the shape (the curvature) of the observed trend in the fitting interval. Finally, in any finite data set there will be some sample variance; i.e., a different draw of the same number of measurements from the same underlying trend, with the same errors, will produce slightly different results. The effects of the sample variance are minimized by having a larger number of data points and can be estimated numerically for any given sample.

We choose a simple powers-of- $z$ polynomial approach, to be maximally model independent. In principle, other basis functions could be used, but we do not see any advantages of such an approach in a situation in which the fits would be dominated by the noise and sparse sampling of the data. We always fit to $y(z)$ and then derive the first and second derivatives from the fit coefficients, and the local values of $E(z)$ and $q(z)$ by using equations (5) and (6). Uncertainties of the fit coefficients are then propagated to derive the uncertainties in the fit values of $y(z), E(z)$, and $q(z)$. The fit values are always evaluated on a redshift grid equally and densely spaced in either $z$ or $\log z$; this is just a matter of convenience, as the values and the quality of the fits are not affected.

The conceptually simplest approach would be to fit a polynomial to the entire data set. Unfortunately, low-order polynomials lack the flexibility to represent the actual shapes of underlying cosmological models, leading to seriously biased values of $E(z)$ and $q(z)$. The fits are (by design) optimized to fit the function $(y)$, and its derivatives are not constrained directly. Using higher order polynomials helps 
in recovering the mean shapes of these functions, but at the expense of greatly increased uncertainties, typically with some oscillatory behavior, characteristic of high-order polynomial fits. For example, the $q(z)$ is generally a nonlinear function of $z$, so the fits of order greater than 3 are needed; but in some cases, e.g., $\Omega_{m}=1$ and $\Omega_{\Lambda}=0$ cosmology, $q(z)=$ const, which higher order polynomials cannot reproduce very easily in shape, regardless of the increased errors for high-order terms.

A better method, which we adopted, is to fit the values of $y(z)$ locally, in some limited redshift window of $\pm \Delta z$; within that interval data points are fitted with the weights inversely proportional to the squares of their error bars. In addition, at each end of the fitting window we attach a Gaussian tapered region with a $\sigma(z)=0.02$, extending out to $2 \sigma$; the enclosed data points in the tapered region have the weights reduced by the value of the Gaussian wing at that point. The purpose of this taper is to avoid fluctuations caused by individual data points entering and leaving the fitting window, at the expense of a slight increase in the resulting fit uncertainties (since the tapered points effectively get larger error bars). We established that the overall properties of the fits did not change. Finally, we require at least 10 data points in each fit and increment the window slightly if necessary.

The trade-off in this technique is that larger fitting windows lead to more robust fits, at the expense of resolution in redshift and the introduction of the same problems that plague the global polynomial fits as described above, while smaller fitting windows produce noisier fits because of a smaller number of enclosed data points. After some experimentation, we concluded that windows with $\Delta z \approx 0.4$ seem to offer the optimal compromise, but we also perform fits with other window sizes.

After some experimentation, we decided to fit secondorder polynomials in each fitting window as the minimalassumption functions with defined second derivatives [needed to evaluate the $q(z)$ ], which can also accomodate any curvature in the data. We verified that using linear fits to obtain $y^{\prime}(z)$ and thus $E(z)$ does not improve results and that increasing the local fit order to 3 increases the formal errors without any significant benefits in terms of the fit quality and accuracy.

Specific details of the fitting procedure are as follows. Let the input data be $\left(z_{i}, y_{i}, \Delta y_{i}\right)$. The fitting weights are computed in a standard fashion as $1 / \Delta y_{i}^{2}$. The fits are evaluated on an output redshift grid $z_{j}$, typically ranging from 0.01 to 1.7 , with a spacing of 0.01 or 0.005 . This is simply a choice of convenience, since the fits can be evaluated anywhere in the redshift regime covered by the data, and there is no reason to do it, say, just at the values of the input $z_{i}$. We note that since our fitting windows $\Delta z$ are generally much larger than the output grid spacing, the adjacent output fit values are not independent. For each output point, $z_{j}=z_{0}$, we select the input data in the corresponding fitting window as described above, ranging from some $i=i_{\min }$ to some $i=i_{\text {max }}$; the fitting weights for points in the Gaussian taper regions are adjusted appropriately. We perform the "centered" fits by introducing the independent variable $x_{i}=z_{i}-z_{0}$ and fit second-order polynomials to $y(x)$ in the range from $i_{\min }$ to $i_{\max }$. We use the routine FIT from Numerical Recipes (Press et al. 1992). The output consists of the fitting coefficients $A, B$, and $C$, where $y(x)=A+B x+C x^{2}$. Then at the centered fit value of $x=0$, the best-fit value for $y(z)=A$, the best-fit value of its first derivative is $d y / d z \equiv d y / d x=B$, and the best-fit value of the second derivative is $d^{2} y / d z^{2} \equiv d^{2} y / d x^{2}=2 C$; thus, from equation (5), $E(z)=1 / B$, and from equation (6), $q(z)=-1-(1+z) 2 C / B$. The routine FIT also returns the covariance matrix, whose diagonal elements give the uncertainties of the coefficients $A, B$, and $C$, and their uncertainties are easily propagated to the fitting uncertainties of $E(z)$ and $q(z)$.

We emphasize that our goal is not to evaluate a number of independent measurements of $E(z)$ and $q(z)$ in the redshift range of interest (the data in hand are not sufficient to do that for an interesting number of points) but instead to outline the global trends presented by the data; thus, we use relatively broad fitting windows with our sliding window fit methodology. The price we pay is the strong correlation of fitted values on our densely spaced output grid, and these should not be taken as independent measurements but really as outlines of the global trends. In our modelindependent approach there is an implicit (and reasonable) assumption that the function $y(z)$ is smoothly changing, with only a modest local curvature. This implies that there is useful "nonlocal" information present in the data, which is captured by our extended fitting windows, at a price of having only two or three "independent" measurements across our full redshift range. With a richer input data set we could make the sliding windows smaller and increase the number of " independent" measurements of $E(z)$ and $q(z)$ (i.e., our redshift resolution of the measured trends).

An alternative approach would be to bin the data in a modest number of redshift bins and perform an independent fit in each bin. An advantage of such a technique would be a set of truly independent measurements of $E(z)$ and $q(z)$, but at the price of increased noise, since the information present by points outside each bin boundary would be lost. This would also lead to implied discontinuities in the fit values of $y(z)$ at the bin edges, leading to implied infinite derivatives, which is obviously unphysical; thus, we opt for a sliding window fit approach, which is essentially a flexible and robust smoothing technique, with a clear caveat that the output fit values are not independent within the used $\Delta z$ range.

In exploring the numerical fitting and differentiation methodology, we used artificial data sets with known builtin cosmologies to evaluate the accuracy of the derived fits for $E(z)$ and $q(z)$. For the most part, we generated artificial data sets mimicking what is expected from SN measurements by the $S N A P$ satellite $^{1}$ (see, e.g., Aldering et al. 2003), namely, a set of 2000 measurements in the redshift interval from 0.1 to 1.7 , with a combined (measurement plus intrinsic) scatter of $7 \%$ in dimensionless coordinate distances. The redshift distribution function was taken to be proportional to the volume element divided by the redshift, which roughly represents a combination of the expected $\mathrm{SN}$ rate history and the $S N A P$ selection function. For most tests, we assumed the standard Friedmann-Lemaitre cosmology with $\Omega_{m}=0.3$ and $\Omega_{\Lambda}=0.7$. For some tests we changed some of these assumptions (the number of the data points, the relative errors, or the underlying cosmology).

The overall process is illustrated in Figure 3, in an example of our pseudo-SNAP data set. The global trends for $y(z), E(z)$, and $q(z)$ are reproduced well, with the bias

\footnotetext{
${ }^{1}$ See also http://snap.lbl.gov.
} 

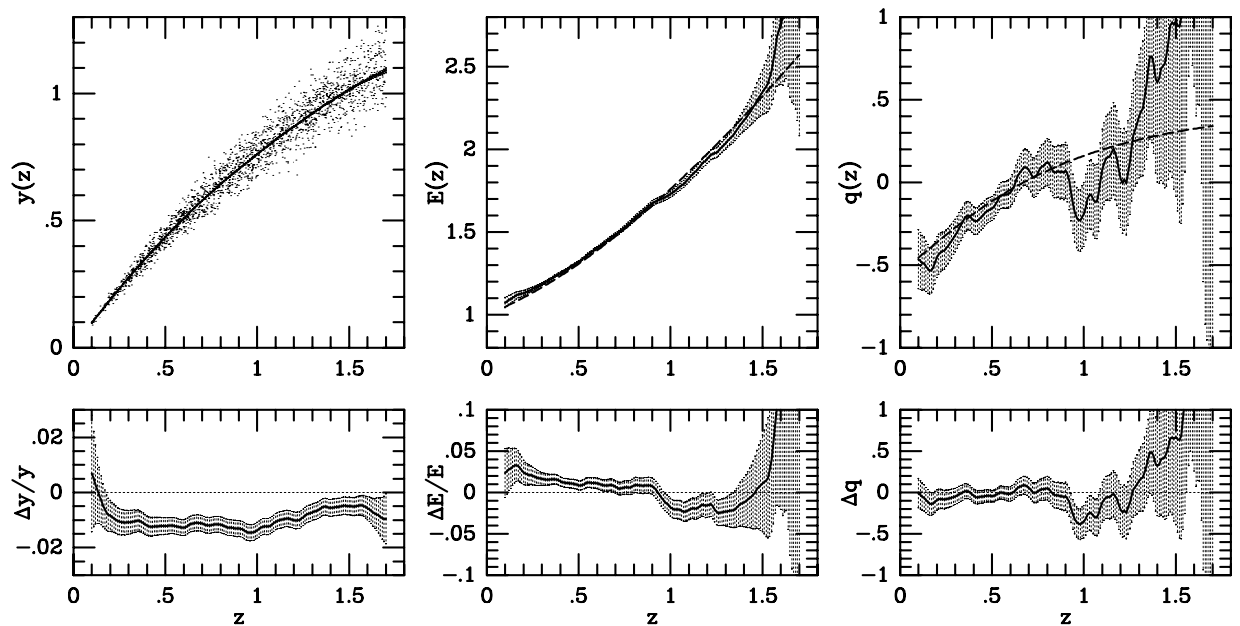

Fig. 3.-Example of the sliding window fitting results for a pseudo-SNAP data set with 2000 data points. We assumed the cosmology with $\Omega_{m}=0.3$ and $\Omega_{\Lambda}=0.7$ and the relative errors $\Delta y / y$ drawn from a Gaussian distribution with $\sigma=7 \%$. A window function with $\Delta z=0.4$ was used. Top left, input data (dots), along with the recovered $y(z)$ trend; bottom left, $y(z)$ fit residuals from the values corresponding to the assumed cosmology; middle, fits for $E(z)$ (top) and its residuals (bottom); right, equivalent for $q(z)$. In all cases the thick line shows the fit values, and the hatched area indicates the $\pm 1 \sigma$ uncertainties. At the top middle and right, the dashed lines show the theoretical values for the assumed cosmology.

(systematic offsets) comparable to the fit errors. The errors increase going toward the higher derivatives and near the edges of the redshift intervals, as may be expected.

The effects of different fitting windows on the derived values $y(z)$ and $E(z)$ are illustrated in Figure 4. As expected, smaller values of $\Delta z$ lead to noisier fits, but the overall trends agree within the errors. For the present $\mathrm{RG}+\mathrm{SN}$ data set, we use $\Delta z$ of 0.4 or 0.6 in what follows.

Finally, we address the issue of the sample variance. For our pseudo-SNAP data, we simply generate a number of different realizations of the data set, using the same assumptions. To estimate the effects for our $\mathrm{RG}+\mathrm{SN}$ data set, we do the following. We assume an underlying cosmology, viz., $\Omega_{m}=0.3$ and $\Omega_{\Lambda}=0.7$. Then, we replace each $y(z)$ measurement with the value for this cosmology, perturbed by a random amount drawn from a Gaussian distribution with the $\sigma(y)$ equal to the quoted error bar. We generate a number of such pseudo-RG+SN data sets and perform the fits on each. The results are shown in Figure 5. For the RG+SN data set, the sample variance effects are comparable to or smaller than the measurement errors for $y(z)$ and comparable to the fitting uncertainties (which derive from the random errors of the data) for $E(z)$. As expected, the sample variance effects for the pseudo-SNAP data set, which has many more data points, are effectively negligible.

Estimates of the sample variance errors are made only rarely in the published literature. Our tests suggest that for many real-life data sets in cosmology, these errors can be easily compared with the fitting uncertainties that derive from the random measurement errors, and thus in many cases the quoted confidence intervals may be underestimating the total uncertainties.

\section{INITIAL RESULTS FOR $E(z)$ AND $q(z)$}

By using the procedure described above, the function $E(z)$ was obtained for the full $\mathrm{RG}+\mathrm{SN}$ data set by using equation (5) and is shown in Figure 6. We used the values of $y_{j}$ for RGs listed in Table 1, and the values for $y$ for $\mathrm{SNe}$

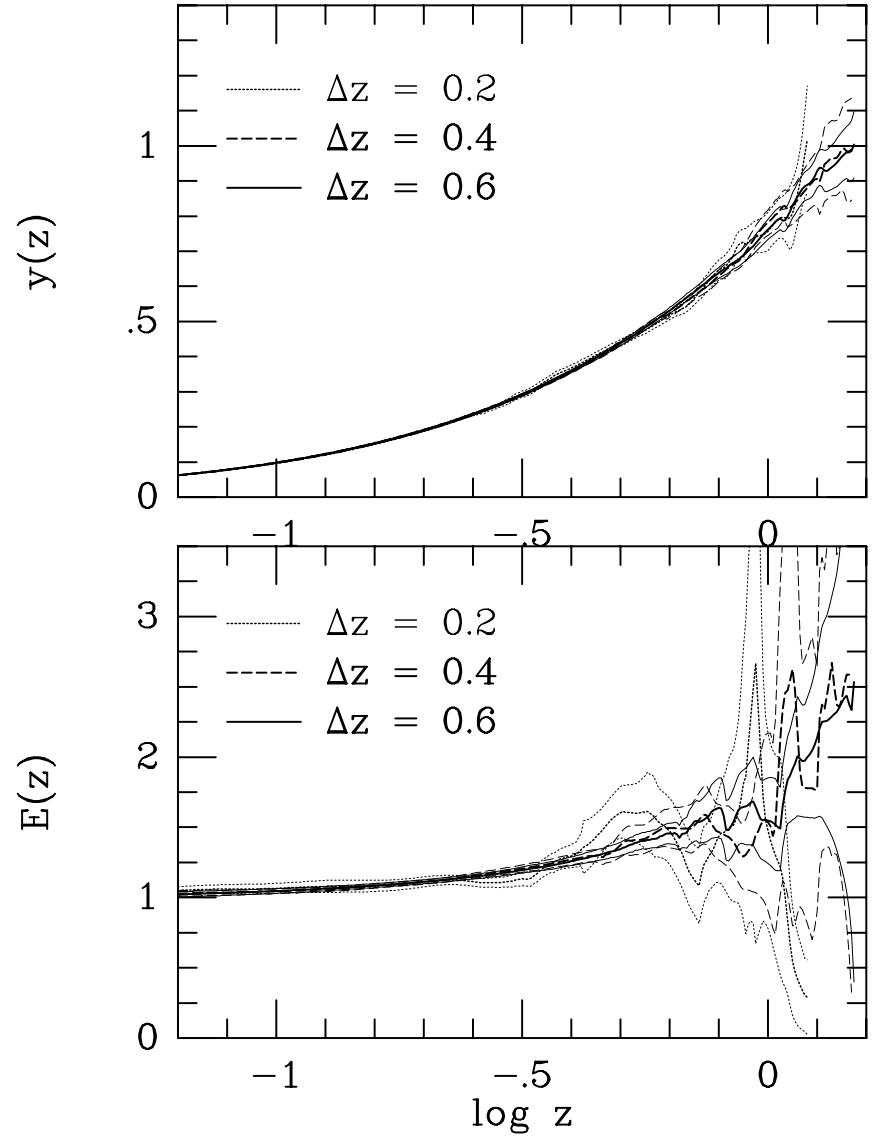

FIG. 4.-Comparison of results obtained for $E(z)$ when different fitting window functions are applied to the full data set of 20 radio galaxies and 78 supernovae. Values of $y_{j}$ for the radio galaxies were used for these fits. Examples of three different window functions are shown with different line types, as indicated. The fit values are indicated by the thick lines, whereas the corresponding thin lines indicate the $\pm 1 \sigma$ range. 

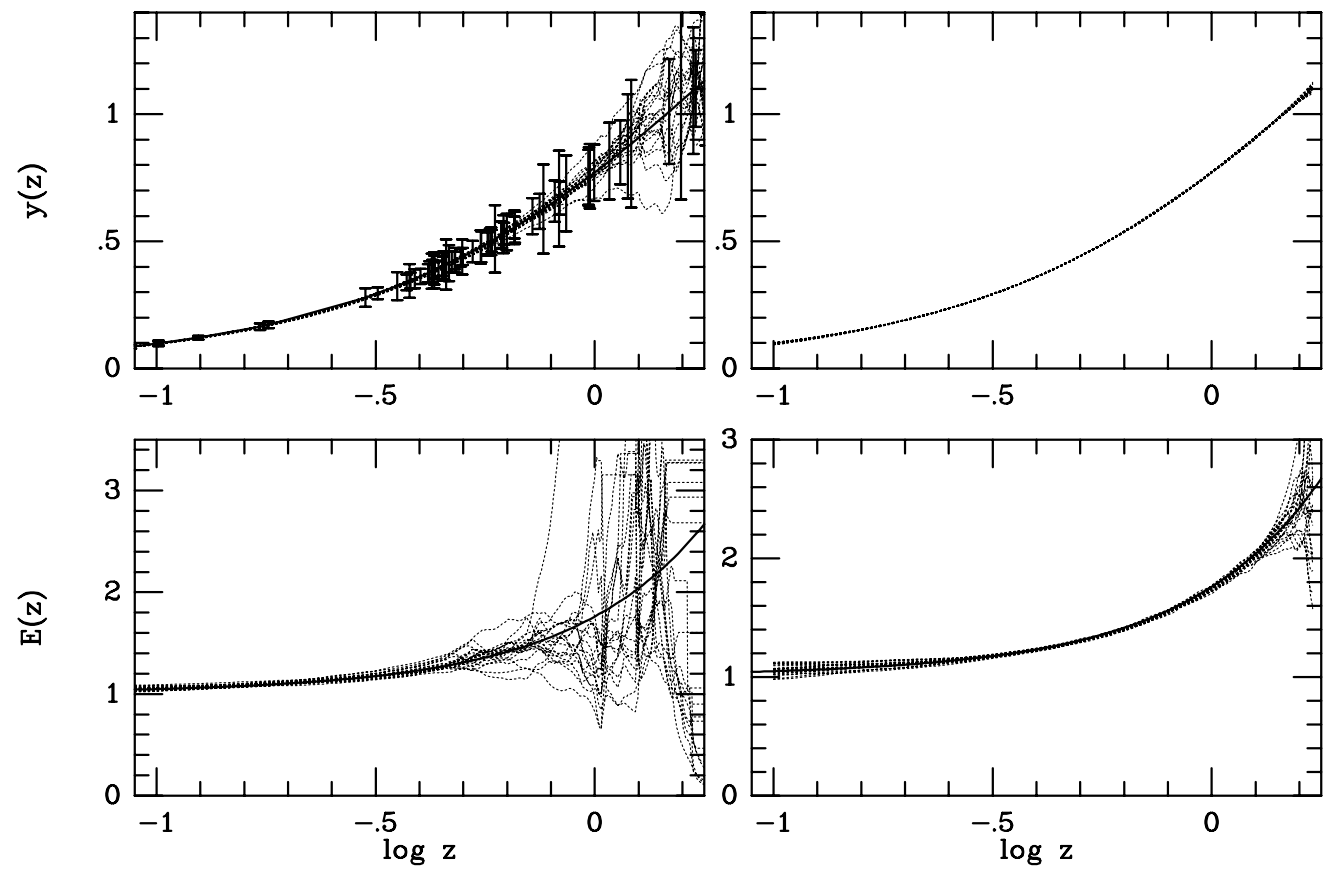

FIG. 5.- Modeling of the sample variance effects for the RG+SN sample (left $)$ and the pseudo-SNAP sample $(r i g h t)$. Values of $y_{j}$ were used for the radio galaxies. A cosmology with $\Omega_{m}=0.3$ and $\Omega_{\Lambda}=0.7$ was assumed (solid lines). The placement and the error bars of the $\mathrm{SN}+\mathrm{RG}$ data points are also indicated at top left. Each dotted line represents a fit from a single random realization of the mock data sets, as described in the text. Their spread at a given redshift is indicative of the sample variance errors. These are obviously much more significant for the smaller sized $N \mathrm{RG}+\mathrm{SN}$ data set than for the much larger pseudoSNAP data set.

listed in Tables 3 and 4; a total of $78 \mathrm{SNe}$ were used, including the average values of $y$ for the $14 \mathrm{SNe}$ listed in Table 4 and values of $y$ for the remaining $64 \mathrm{SNe}$ listed in Table 3. The results are remarkably close to the currently popular "concordance" Friedmann-Lemaitre model with $\Omega_{\Lambda}=0.7$ and $\Omega_{m}=0.3$. We note, however, that in our analysis we did not assume that the universe is described by a FriedmannLemaitre model at all.

As an internal consistency test, we computed the fits for the RG and SN samples separately, using the values $y_{s}$ for the radio galaxies. The results are shown in Figure 7 . It is

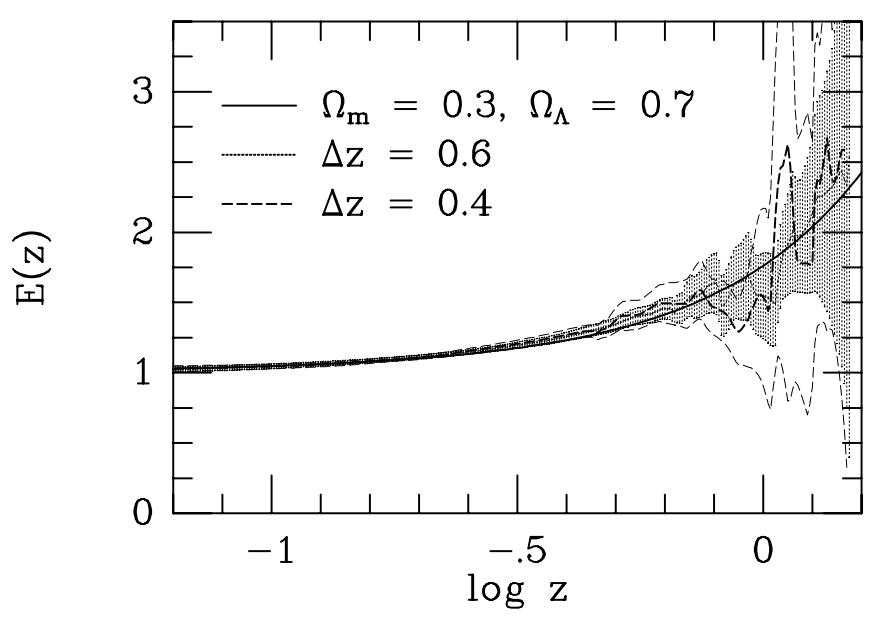

FIG. 6.-First look at $E(z)$ for the full data set, with values of $y_{j}$ used for the radio galaxies, showing fits with $\Delta z=0.6$ (thick dotted line), $\pm 1 \sigma$ range (dotted hatched area), fits with $\Delta z=0.4$ (thick dashed line), $\pm 1 \sigma$ range (thin dashed lines), and the trend for the $\Omega_{m}=0.3$ and $\Omega_{\Lambda}=0.7$ Friedmann-Lemaitre cosmology (thick solid line). clear that the values of $y$ obtained for radio galaxies and supernovae agree very well for the redshifts in which the data sets overlap, and in the redshift range in which the two samples overlap the independent determinations of $E(z)$ agree to within $1 \sigma$ (joint errors) or better. It is notable that the RG data alone are consistent with a constant $E(z) \approx 1.1$ for $z \sim 0.4-1.8$, although the error bars are large. If this trend remains as the error bars decrease with better and more extensive data sets, it could be indicative of an actual cosmological trend or (perhaps more likely) some evolutionary effect or bias in the data. This cannot be sorted out until more data are available.

Finally, we show in Figure 8 what is probably the first direct estimate of $q(z)$, obtained using equation (6). The data, folded through our analysis procedure, are fully consistent with the "concordance" model with $\Omega_{\Lambda}=0.7$ and $\Omega_{m}=0.3$ and suggest that indeed the universe makes a transition from deceleration to acceleration at $z_{T} \gtrsim 0.3$, with a best-fit value of $z_{T} \approx 0.45$. Again, we note that no assumptions about the cosmological model have been made in deriving this trend. This is a preliminary result, and we are clearly limited by the available data at this time. Our purpose here is mainly to illustrate the method, but even so the results are very encouraging.

\section{IMPLICATIONS OF $E(z)$ AND $q(z)$ FOR THE PROPERTIES OF THE DARK ENERGY}

The acceleration parameter is $q(z)=-\ddot{a} a / \dot{a}^{2}=$ $-(\ddot{a} / a)(\dot{a} / a)^{-2}$. The acceleration of the universe is described by

$$
\frac{\ddot{a}}{a}=-\frac{4 \pi G}{3} \sum\left(\rho_{i}+3 p_{i}\right)=-\frac{4 \pi G}{3} \sum \rho_{i}\left(1+3 w_{i}\right),
$$


TABLE 3

Supernova Ia Dimensionless Coordinate Distances

\begin{tabular}{|c|c|c|c|c|}
\hline Source & Redshift & $y_{s}$ & $\sigma\left(y_{s}\right)$ & Reference \\
\hline 1996C ............... & 0.009 & 0.008 & 0.0012 & 1 \\
\hline 1995D ............... & 0.012 & 0.011 & 0.0011 & 1 \\
\hline 1992al ................ & 0.014 & 0.013 & 0.0013 & 2 \\
\hline 1992al ................. & 0.014 & 0.013 & 0.0009 & 1 \\
\hline 1995ak ............... & 0.016 & 0.015 & 0.0014 & 1 \\
\hline 1994S ................ & 0.016 & 0.015 & 0.0010 & 1 \\
\hline 1992bo ................ & 0.018 & 0.019 & 0.0016 & 1 \\
\hline $1992 b c$............... & 0.020 & 0.017 & 0.0016 & 2 \\
\hline $1992 b c$............... & 0.020 & 0.020 & 0.0012 & 1 \\
\hline 1995 ac................. & 0.022 & 0.023 & 0.0018 & 1 \\
\hline 1994M ............... & 0.024 & 0.026 & 0.0022 & 1 \\
\hline 1993Н .............. & 0.025 & 0.024 & 0.0029 & 1 \\
\hline 1992ag .............. & 0.026 & 0.026 & 0.0028 & 1 \\
\hline $1992 \mathrm{ag} \ldots \ldots \ldots \ldots \ldots$ & 0.026 & 0.029 & 0.0027 & 2 \\
\hline 1992P ................. & 0.026 & 0.026 & 0.0029 & 2 \\
\hline 1992 Р ........ & 0.026 & 0.030 & 0.0020 & 1 \\
\hline 1995bd ............... & 0.028 & 0.034 & 0.0033 & 1 \\
\hline 19990 ................ & 0.030 & 0.028 & 0.0026 & 2 \\
\hline $1992 b g \ldots \ldots \ldots \ldots$ & 0.035 & 0.038 & 0.0038 & 1 \\
\hline 1994T ................. & 0.036 & 0.035 & 0.0035 & 1 \\
\hline $1992 b g \ldots \ldots \ldots \ldots . . . . .$. & 0.036 & 0.034 & 0.0033 & 2 \\
\hline $1992 \mathrm{bl} \mathrm{f..............}$ & 0.043 & 0.043 & 0.0036 & 2 \\
\hline $1992 b 1 \ldots \ldots \ldots \ldots \ldots$ & 0.043 & 0.038 & 0.0026 & 1 \\
\hline 1992bh ............... & 0.045 & 0.051 & 0.0040 & 1 \\
\hline 1992bh .............. & 0.045 & 0.052 & 0.0046 & 2 \\
\hline 1995E................. & 0.049 & 0.049 & 0.0031 & 1 \\
\hline 1990af ................. & 0.050 & 0.052 & 0.0044 & 2 \\
\hline 1993ag ................ & 0.050 & 0.054 & 0.0050 & 2 \\
\hline 1993ag ............... & 0.050 & 0.048 & 0.0038 & 1 \\
\hline 1990af ................ & 0.050 & 0.042 & 0.0031 & 1 \\
\hline 19930 ................ & 0.052 & 0.053 & 0.0030 & 1 \\
\hline $19930 \ldots \ldots \ldots \ldots \ldots$ & 0.052 & 0.050 & 0.0042 & 2 \\
\hline 1992bs............... & 0.063 & 0.068 & 0.0057 & 2 \\
\hline 1992bs................ & 0.064 & 0.069 & 0.0045 & 1 \\
\hline 1993B................ & 0.071 & 0.071 & 0.0065 & 2 \\
\hline 1992ae.................. & 0.075 & 0.074 & 0.0058 & 1 \\
\hline 1992ae................ & 0.075 & 0.074 & 0.0068 & 2 \\
\hline 1992bp ............. & 0.079 & 0.068 & 0.0057 & 2 \\
\hline 1992bp ............. & 0.080 & 0.069 & 0.0044 & 1 \\
\hline 1992 br............... & 0.087 & 0.087 & 0.0077 & 1 \\
\hline $1992 \mathrm{aq} \ldots \ldots \ldots \ldots \ldots$ & 0.101 & 0.096 & 0.0067 & 1 \\
\hline $1992 \mathrm{aq} \ldots \ldots \ldots \ldots . . . .$. & 0.101 & 0.101 & 0.0107 & 2 \\
\hline 1996ab ............... & 0.124 & 0.124 & 0.0073 & 1 \\
\hline 1997I................. & 0.172 & 0.151 & 0.0126 & 2 \\
\hline 1997N ................ & 0.180 & 0.169 & 0.0133 & 2 \\
\hline 1996J ................ & 0.300 & 0.319 & 0.0357 & 1 \\
\hline 1997ac................ & 0.320 & 0.291 & 0.0243 & 2 \\
\hline 1994F ................ & 0.354 & 0.361 & 0.0549 & 2 \\
\hline 1994am ............... & 0.372 & 0.337 & 0.0312 & 2 \\
\hline 1994an ................. & 0.378 & 0.389 & 0.0663 & 2 \\
\hline 1996K ............... & 0.380 & 0.337 & 0.0310 & 1 \\
\hline 1995ba .............. & 0.388 & 0.399 & 0.0369 & 2 \\
\hline 1995aw............... & 0.400 & 0.346 & 0.0304 & 2 \\
\hline 1997am ............... & 0.416 & 0.376 & 0.0349 & 2 \\
\hline 1994al ................. & 0.420 & 0.372 & 0.0430 & 2 \\
\hline 1994G ................ & 0.425 & 0.305 & 0.0690 & 2 \\
\hline 1996Е................ & 0.425 & 0.344 & 0.0449 & 1 \\
\hline 1997Q ................ & 0.430 & 0.373 & 0.0311 & 2 \\
\hline 1996U ................. & 0.430 & 0.497 & 0.0574 & 1 \\
\hline 1997ce............... & 0.440 & 0.375 & 0.0304 & 1 \\
\hline 1995az................ & 0.450 & 0.358 & 0.0380 & 2 \\
\hline $1996 \mathrm{~cm}$............... & 0.450 & 0.485 & 0.0515 & 2 \\
\hline 1997ai .................. & 0.450 & 0.414 & 0.0574 & 2 \\
\hline 1995aq ............... & 0.453 & 0.484 & 0.0559 & 2 \\
\hline 1992bi .................. & 0.458 & 0.469 & 0.0994 & 2 \\
\hline
\end{tabular}

TABLE 3-Continued

\begin{tabular}{|c|c|c|c|c|}
\hline Source & Redshift & $y_{s}$ & $\sigma\left(y_{s}\right)$ & Reference \\
\hline 1995ar.................... & 0.465 & 0.516 & 0.0715 & 2 \\
\hline 1997P ....................... & 0.472 & 0.464 & 0.0409 & 2 \\
\hline 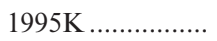 & 0.478 & 0.459 & 0.0353 & 1 \\
\hline 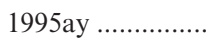 & 0.480 & 0.431 & 0.0478 & 2 \\
\hline 1996сі ................. & 0.495 & 0.402 & 0.0354 & 2 \\
\hline 1995as .................... & 0.498 & 0.602 & 0.0695 & 2 \\
\hline 1997cj .................... & 0.500 & 0.442 & 0.0340 & 1 \\
\hline 1997Н ................. & 0.526 & 0.456 & 0.0423 & 2 \\
\hline 1997L ................... & 0.550 & 0.530 & 0.0613 & 2 \\
\hline 1996cf .................... & 0.570 & 0.469 & 0.0477 & 2 \\
\hline 19961_................... & 0.570 & 0.499 & 0.0430 & 1 \\
\hline 1997af .................... & 0.579 & 0.513 & 0.0522 & 2 \\
\hline 1997F ................... & 0.580 & 0.508 & 0.0541 & 2 \\
\hline 1997aj ................... & 0.581 & 0.428 & 0.0436 & 2 \\
\hline 1997K ….............. & 0.592 & 0.785 & 0.1340 & 2 \\
\hline 1997S …............... & 0.612 & 0.554 & 0.0538 & 2 \\
\hline 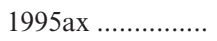 & 0.615 & 0.439 & 0.0507 & 2 \\
\hline 1997J ................... & 0.619 & 0.580 & 0.0750 & 2 \\
\hline 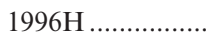 & 0.621 & 0.535 & 0.0434 & 1 \\
\hline 1995at .................... & 0.655 & 0.445 & 0.0432 & 2 \\
\hline 1996ck .................. & 0.656 & 0.510 & 0.0660 & 2 \\
\hline 1997R ….............. & 0.657 & 0.575 & 0.0611 & 2 \\
\hline 1997G .................. & 0.763 & 0.725 & 0.1772 & 2 \\
\hline 1996cl ................... & 0.828 & 0.760 & 0.1892 & 2 \\
\hline 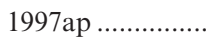 & 0.830 & 0.652 & 0.0664 & 2 \\
\hline 1997ck .................... & 0.970 & 0.844 & 0.1169 & 1 \\
\hline 1997ff .................... & 1.700 & 0.967 & 0.1517 & 3 \\
\hline
\end{tabular}

References.- (1) Riess et al. 1998; (2) Perlmutter et al. 1999; (3) Riess et al. 2003.

where $p_{i}$ is the pressure, $\rho_{i}$ is the mean mass-energy density, and $w_{i}$ is the equation of state of the $i$ th component, $w_{i}=$ $p_{i} / \rho_{i}$. In addition, for $k=0$,

$$
\left(\frac{\dot{a}}{a}\right)^{2}=\frac{8 \pi G}{3} \sum \rho_{i} .
$$

Thus, $q(z)=0.5 \sum \rho_{i}\left(1+3 w_{i}\right) / \sum \rho_{i}$ when $k=0$, and $E^{2}(z)=\sum \rho_{i} / \rho_{c 0}$, where $\rho_{c 0}$ is the critical density at the present epoch, $\rho_{c 0} \equiv(3 / 8 \pi G) H_{0}^{2}=\rho_{m 0}+\rho_{E 0}$, and the present epoch mean mass-energy density of nonrelativistic

TABLE 4

Average Values of $y$ for Supernovae LiSTED BY Both RiESS ET AL. 1998 AND Perlmutter et al. 1999

\begin{tabular}{|c|c|c|c|}
\hline Source & Redshift & $y_{s}$ & $\sigma\left(y_{s}\right)$ \\
\hline 1990af ...... & 0.050 & 0.047 & 0.0053 \\
\hline 1992ae ................... & 0.075 & 0.074 & 0.0090 \\
\hline 1992ag ……......... & 0.026 & 0.027 & 0.0039 \\
\hline 1992al ...................... & 0.014 & 0.013 & 0.0016 \\
\hline 1992aq …............... & 0.101 & 0.098 & 0.0126 \\
\hline $1992 b c$................. & 0.020 & 0.019 & 0.0020 \\
\hline 1992bg .................. & 0.036 & 0.036 & 0.0050 \\
\hline 1992bh .................... & 0.045 & 0.051 & 0.0061 \\
\hline 1992bl ….............. & 0.043 & 0.040 & 0.0044 \\
\hline 1992bp ................ & 0.079 & 0.069 & 0.0072 \\
\hline 1992bs................... & 0.063 & 0.069 & 0.0073 \\
\hline 1992Р ................. & 0.026 & 0.028 & 0.0035 \\
\hline 1993ag ................ & 0.050 & 0.051 & 0.0063 \\
\hline 19930 …............. & 0.052 & 0.052 & 0.0051 \\
\hline
\end{tabular}



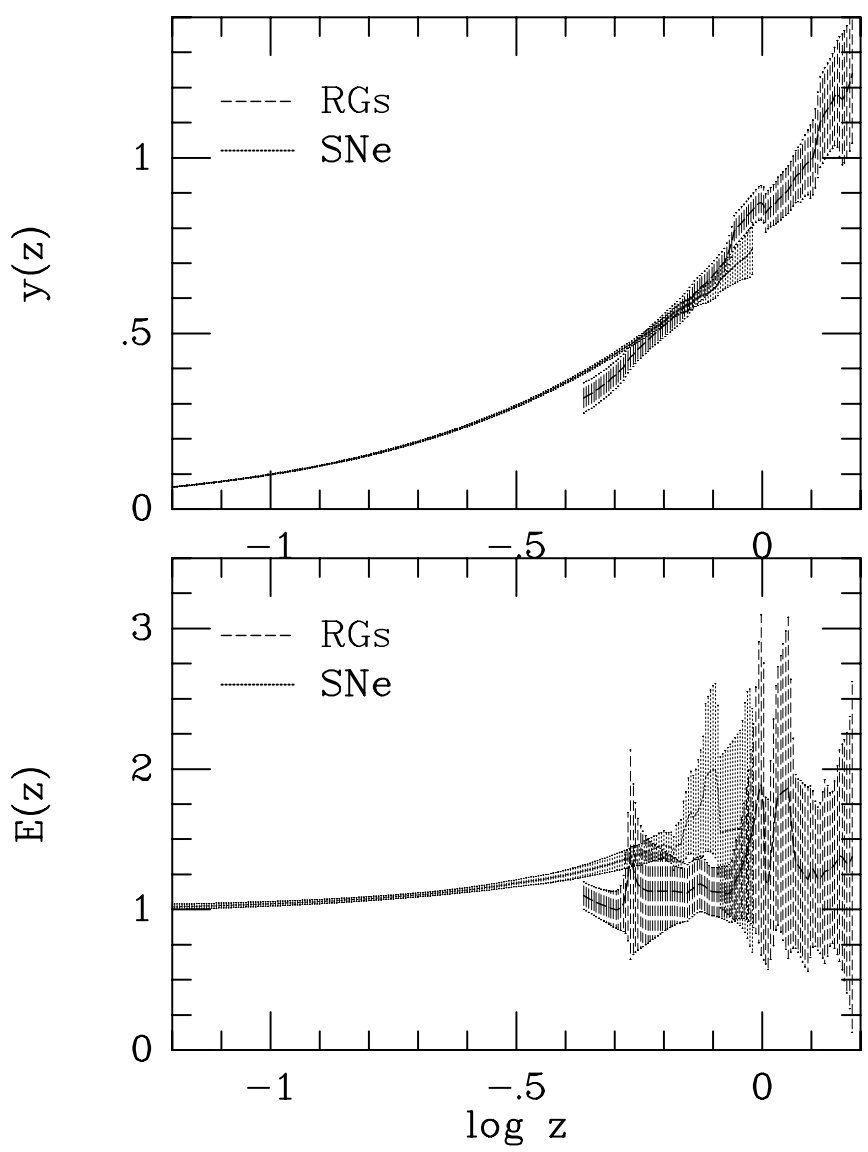

FIG. 7.-Comparison of the fits for $\mathrm{SN}$ and RG data sets computed separately. Values of $y_{s}$ for RGs were used for these fits, showing the $\mathrm{SN}$ data (dotted line) with the best-fit values and the $\pm 1 \sigma$ range (dotted hatched area); and the RG data (dashed line) with the best-fit values and the $\pm 1 \sigma$ range (dashed hatched area).

matter and dark energy are $\rho_{m 0}$ and $\rho_{E 0}$, respectively. Nonrelativistic matter evolves with redshift as $(1+z)^{3}$. Let the dark energy evolve with redshift as $f_{E}(z)$; then, it is easy to show that $E^{2}(z)=\Omega_{m}(1+z)^{3}+\left(1-\Omega_{m}\right) f_{E}(z)$, where $\Omega_{m}=\rho_{m 0} / \rho_{c 0}$ and $\Omega_{E}=\rho_{E 0} / \rho_{c 0}=1-\Omega_{m}$. Hence, if the current contribution of nonrelativistic matter $\Omega_{m}$ can be determined, then $E(z)$ can be used to determine the redshift evolution of the dark energy $f_{E}(z)$. Similarly,

$$
q(z)=0.5\left(\frac{\Omega_{m}+\left(1-\Omega_{m}\right)(1+3 w)(1+z)^{-3} f_{E}(z)}{\Omega_{m}+\left(1-\Omega_{m}\right)(1+z)^{-3} f_{E}(z)}\right) .
$$

Now, for quintessence, $\rho_{i}=\rho_{i, 0}(1+z)_{i}^{n}$ when $w_{i}$ is constant, where $n_{i}=3\left(1+w_{i}\right)$. This follows from the massenergy conservation of each component, which implies

$$
\dot{\rho}_{i}=-3\left(\rho_{i}+p_{i}\right)(\dot{a} / a)
$$

(e.g., Peebles 1993). When the equation of state $w_{i}$ does not change with time, the solution to this equation is $\rho_{i}=$ $\rho_{i, 0}(1+z)^{3\left(1+w_{i}\right)}$, where $(1+z)=a_{0} / a$; thus, a component with equation of state $w_{i}$ and present mean mass-energy density $\rho_{0}$ will have a mean mass-energy density at redshift $z$ of $\rho=\rho_{0}(1+z)^{n_{i}}$, where $n_{i}=3\left(1+w_{i}\right)$.

With two important components at low redshift, a nonrelativistic component $\rho_{m}$ that includes baryons and the dark matter in galaxies and clusters of galaxies, and a dark energy component $\rho_{E}$, we have $\rho_{m}=\rho_{m 0}(1+z)^{3}$ and $\rho_{E}=$

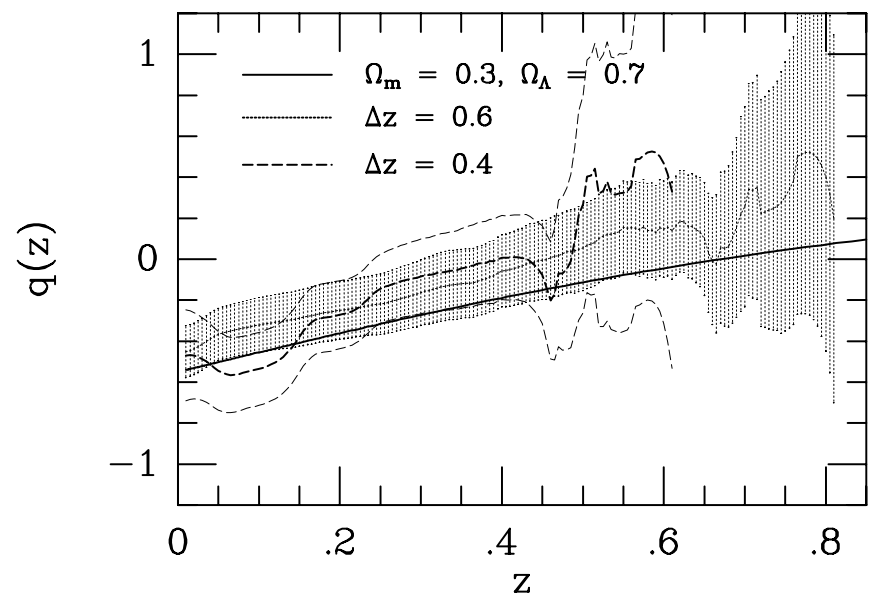

FIG. 8.-First look at $q(z)$, obtained for the full data set, with values of $y_{j}$ used for the radio galaxies, showing fits with $\Delta z=0.6$ (thick dotted line), $\pm 1 \sigma$ range (dotted hatched area); fits with $\Delta z=0.4$ (thick dashed line), $\pm 1 \sigma$ range (thin dashed lines); and the trend for the $\Omega_{m}=0.3$ and $\Omega_{\Lambda}=0.7$ Friedmann-Lemaitre cosmology (thick solid line). The fits become too noisy to be useful past about $z \sim 0.8$ in this data set.

$\rho_{E 0}(1+z)^{n}$. Now, at zero redshift the total density is equal to the critical density $\rho_{c 0}=\rho_{m 0}+\rho_{E 0}$, and since $\rho_{m 0} / \rho_{0 c} \equiv \Omega_{m}$, the acceleration parameter can be written

$$
q(z)=0.5\left(\frac{\Omega_{m}+\left(1-\Omega_{m}\right)(1+3 w)(1+z)^{3 w}}{\Omega_{m}+\left(1-\Omega_{m}\right)(1+z)^{3 w}}\right) .
$$

The universe is decelerating when the sign of $q(z)$ is positive. The sign of the denominator is always positive, and the numerator may be either positive or negative, depending on the value of $w$. The universe will go from a state of acceleration to a state of deceleration if the dark energy has properties like that of quintessence when the numerator of equation (11) is positive, which occurs at the transition redshift $z_{T}$ given by

$$
z_{T}=\left[\frac{-\Omega_{m}}{(1+3 w)\left(1-\Omega_{m}\right)}\right]^{1 /(3 w)}-1 .
$$

This transition redshift is plotted as a function of the equation of state $w$ in Figure 9. Clearly, as the transition redshift increases, the value of $\Omega_{m}$ must decrease, or the equation of state $w$ must exhibit redshift evolution.

Similarly, for quintessence, $E^{2}(z)=(1+z)^{3}\left[\Omega_{m}+\right.$ $\left.\left(1-\Omega_{m}\right)(1+z)^{3 w}\right]$. Some lines representing quintessence with $w=-1$ (i.e., a cosmological constant) are included in the figures.

Our preliminary results set a limit on the transition redshift $z_{T} \gtrsim 0.3$, with the best-fit estimate $z_{T} \approx 0.45$. Assuming $\Omega_{m}=0.3$, these translate to $w \lesssim-0.55$ and $-2.3 \lesssim w \lesssim-0.65$ (see Fig. 9). With better data sets in the future, we should be able to improve on these limits.

Note that $q(z)$ is an important input into the Statefinder diagnostic presented by Sahni et al. (2003) and Alam et al. (2003); these authors discuss one way in which $q(z)$ may be used to determine $w(z)$ and the Statefinder pair $(r, s)$.

\section{SUMMARY AND DISCUSSION}

We presented here a set of dimensionless coordinate distances for $20 \mathrm{RGs}$, spanning the redshift range 0.43-1.79 (with one source at $z=0.056$ ). These measurements 


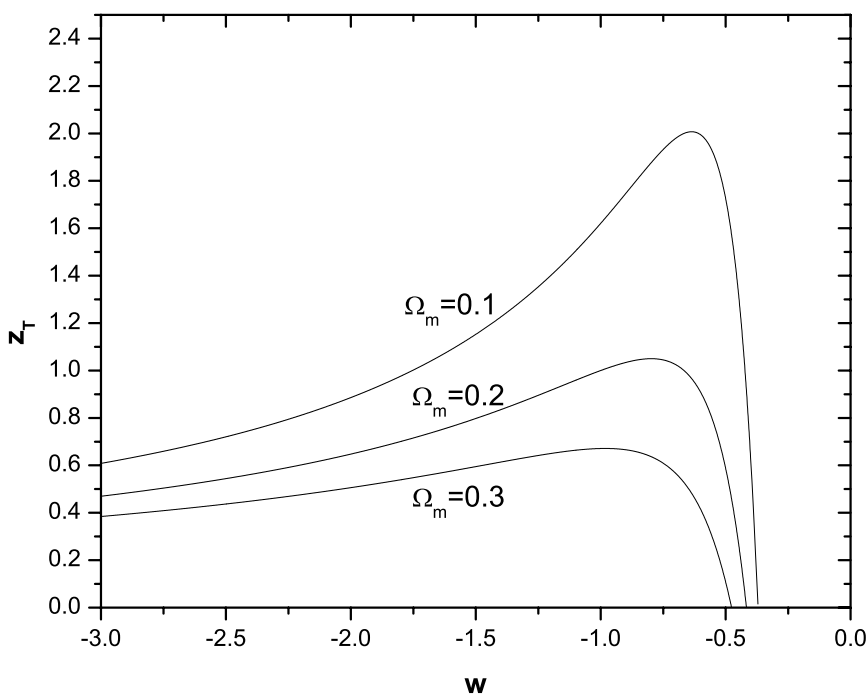

FIG. 9.-Transition redshift, $z_{T}$, at which the universe makes a transition from a state of deceleration to a state of acceleration, as a function of the equation of state of the dark energy, assuming that the equation of state of the dark energy is time independent. If the empirically determined $q(z)$ limits the transition redshift, then bounds can be placed on the amount and redshift evolution of the dark energy.

supplement and extend to the cosmologically interesting redshift range the distances available for $\mathrm{SNe}$, which currently reach only to $z=0.97$ (with one source at $z=1.70$ ).

The determination of the dimensionless coordinate distances to RGs and $\mathrm{SNe}$ are completely independent and are based on completely different physics. Yet, the two data sets agree very well in the overlap redshift range, as shown here and as shown by previous work (e.g., Podariu et al. 2003; Daly \& Guerra 2002; Guerra et al. 2000). This is very encouraging: there is a great value in being able to measure the same physical quantity (here the coordinate distances as a function of redshift) using different and independent tracers and methods. The general agreement we see between the SN and RG data sets suggests that neither method is dominated by some substantial, as yet unknown, systematic error. Together, the two data sets can be used in cosmological tests with greater power than each data set separately.

The dimensionless coordinate distances $y(z)$ can be used to empirically determine the dimensionless expansion and deceleration rates as functions of redshift, $E(z)$ and $q(z)$, without assuming any particular cosmological model. While the traditional cosmological tests integrate the expressions for these functions provided by the models (e.g., the standard Friedmann-Lemaitre models) and determine the model parameters from the fits, we develop a complementary procedure whereby these functions can be derived directly from the data by differentiating the $y(z)$ trend. We apply a particular simple numerical procedure to this task and derive the trends of both $E(z)$ and - for the first time$q(z)$ directly from the data.

Our estimates of $E(z)$ are in excellent agreement with those obtained from other methods, e.g., the CMB fluctuations, large-scale structure, high- $z \mathrm{SNe}$ and radio galaxies by using traditional analyses, etc., even though they are obtained in a completely different and independent manner. In particular, the data are consistent with the "concordance" cosmology, i.e., Friedmann-Lemaitre models with $\Omega_{m}=0.3$ and $\Omega_{\Lambda}=0.7$. While these results are clearly very preliminary and meant primarily to illustrate the method, the good agreement with other approaches is very encouraging.

We are currently limited by the amount and quality of the available data for both SNe and RGs. Nevertheless, there are great prospects for advances in precision cosmology, e.g., large sets of high-quality measurements of $\mathrm{SNe}$ from the $S N A P$ satellite (e.g., Aldering et al. 2003) or from large ground-based experiments such as ESSENCE ${ }^{2}$ (Stubbs 2002) or LSST in the future (Tyson et al. 2003a, 2003b). Such data sets could certainly support differentiation of distance versus redshift trends, leading to considerably more robust direct determinations of the expansion and acceleration rates as functions of redshift. In addition, new radio galaxy data are being obtained.

As the observational situation improves, direct estimates of $E(z)$ and $q(z)$ can be used to understand the properties and redshift evolution of different flavors of dark energy and determine the redshift at which the universe makes a transition from acceleration to deceleration, and they may help elucidate any systematic errors that might be lurking in the $\mathrm{RG}$ or $\mathrm{SN}$ methods of constraining cosmological parameters.

It is a pleasure to thank Megan Donahue, Eddie Guerra, Matt Mory, Chris O’Dea, Paddy Padmanabhan, Bharat Ratra, and Varun Sahni for helpful comments and discussions, Saul Perlmutter for sending electronic files of supernova data, and Adam Riess for providing us with supernova data modified to be on the same scale as that provided by Saul Perlmutter. This work was supported in part by the National Science Foundation under grants AST 00-96077 and AST 02-06002, by Pennsylvania State University (R. A. D.), and by the Ajax Foundation (S. G. D).

\footnotetext{
${ }^{2}$ See also http://www.ctio.noao.edu/wproject.
}

\section{REFERENCES}

Alam, U., Sahni, V., Saini, T. D., \& Starobinsky, A. A. 2003, MNRAS, 344,1057

Aldering, G., et al. 2003, Proc. SPIE, 4835, 146

Armendariz-Picon, C., Damour, T., \& Mukhanov, V. 1999, Phys. Lett. B, 458, 209

Astier, P. 2001, Phys. Lett. B, 500, 8

Barger, V., \& Marfatia, D. 2001, Phys. Lett. B, 498, 67

Benitez, N., Riess, A., Nugent, P., Dickinson, M., Chornock, R., \& Filippenko, A. V. 2002, ApJ, 577, L

Bennett, C., et al. 2003, ApJ, 583, 1

Buchalter, A., Helfand, D. J., Becker, R. H., \& White, R. L. 1998, ApJ, 494,503

Caldwell, R. R., Dave, R., \& Steinhardt, P. J. 1998, Phys. Rev. Lett., 80, 1582

Carroll, S. M., Press, W. H., \& Turner, E. L. 1992, ARA\&A, 30, 499

Chen, G., \& Ratra, B. 2003, ApJ, 582, 586
Chiba, T., \& Nakamura, T. 2000, Phys. Rev. D, 62, 121301

Daly, R. A. 1994, ApJ, 426, 38

. 2002, in Particle Astrophysics and Cosmology: From Quarks to the Cosmos, ed. T. Sumner (Amsterdam: Elsevier), in press (astro-ph/ 0212107)

Daly, R. A., \& Guerra, E. J. 2002, AJ, 124, 1831

Frampton, P. H. 2003, Phys. Lett. B, 555, 139

Gerke, B. F., \& Efstathiou, G. 2002, MNRAS, 335, 33

Goliath, M., Amanullah, T., Astier, P. Goobar, A., \& Pain, R. 2001, A\&A, 380,6

Guerra, E. J., \& Daly, R. A. 1998, ApJ, 493, 536

Guerra, E. J., Daly, R. A., \& Wan, L. 2000, ApJ, 544, 659

Gurvits, L. I., Kellermann, K. I., \& Frey, S. 1999, A\&A, 342, 378

Huterer, D., \& Turner, M. S. 1999, Phys. Rev. D, 60, 081301

Leibundgut, B. 2001, ARA\&A, 39, 67

Lima, J. A. S., \& Alcaniz, J. S. 2002, ApJ, 566, 15 
Maor, I., Brustein, R., \& Steinhardt, P. 2001, Phys. Rev. Lett., 86, 6

Padmanabhan, T. 2003, Phys. Rep., in press

Padmanabhan, T., Choudhury, T. R. 2002, MNRAS, 344, 823

Peebles, P. J. E. 1993, Principles of Physical Cosmology (Princeton: Princeton Univ. Press)

Peebles, P. J. E., \& Ratra, B. 1988, ApJ, 325, L17

2003, Rev. Mod. Phys., 75, 559

Perlmutter, S., et al. 1999, ApJ, 517, 565

Podariu, S., Daly, R. A., Mory, M. P., \& Ratra, B. 2003, ApJ, 584, 577

Press, W., Teukolsky, S., Vetterling, W., \& Flannery, B. 1992, Numerical

Recipes (2d ed.; Cambridge: Cambridge Univ. Press)

Riess, A. 2000, PASP, 112, 1284

Riess, A., et al. 1998, AJ, 116, 1009 2001, ApJ, 560, 49

2003, PASP, in press

Sahni, V., Saini, T. D., Starobinsky, A. A., \& Alam, U. 2003, J. Exp. Theor. Phys. Lett., 77, 201

Sahni, V., \& Starobinsky, A. A. 2000, Int. J. Mod. Phys., D9, 373
Saini, T., Raychaudhury, S., Sahni, V., \& Starobinsky, A. A. 2000, Phys. Rev. Lett., 85, 1162

Spergel, D., et al. 2003, ApJS, 148, 175

Starobinsky, A. 1988, Zh. Eksp. Teor. Fiz. Lett., 68, 757

Stubbs, C. 2002, BAAS, 34, 1279

Turner, M. 2002, Int. J. Mod. Phys., A17S1, 180

2003, in ASP Conf Ser. 291, Hubble's Science Legacy: Future Optical/Ultraviolet Astronomy from Space, ed. K. R. Sembach et al. (San Francisco: ASP)

Turner, M. S., \& White, M. 1997, Phys. Rev. D, 56, R4439

Tyson, J. A., Wittman, D., Hennawi, J., \& Spergel, D. 2003a, in Proc. 5th International UCLA Symposium on Sources and Detection of Dark Matter, ed. D. Cline, in press

Tyson, J. A., et al. (LSST). 2003b, Proc. SPIE, 4836, 10

Vishwakarma, R. G. 2001, Classical Quantun Gravity, 18, 1159

Weinberg, S. 1972, Gravitation and Cosmology (New York: Wiley)

Weller, J., \& Albrecht, A. 2002, Phys. Rev. D, 65, 103512 